\title{
LAS LARVAS DEL GÉNERO IDAEA TREITSCHKE, 1825 (INSECTA, LEPIDOPTERA, GEOMETRIDAE): NUEVOS DATOS SOBRE SU MORFOLOGÍA Y BIOLOGÍA
}

\author{
G. E. King ${ }^{1} \&$ J. L. Viejo Montesinos ${ }^{2}$
}

\section{RESUMEN}

G. E. King \& J. L. Viejo Montesinos. 2012. Las larvas del género /daea Treitschke, 1825 (Insecta, Lepidoptera, Geometridae): Nuevos datos sobre su morfología y biología. Graellsia, 68(2): 249-262.

Se ha estudiado la biología y la quetotaxia de las larvas del género /daea Treitschke, 1825 mediante el análisis de las larvas o de las exuvias larvarias de seis especies o subespecies: I. sericeata calvaria Wehrli, 1927, I. ochrata albida (Zerny, 1936), I. incisaria (Staudinger, 1892), I. bigladiata Herbulot, 1975, I. cervantaria (Millière, 1869) e I. degeneraria (Hübner, 1799), junto con datos biológicos de larvas de otras seis especies: I. ochrata (Scopoli, 1763), I. macilentaria (Herrich-Schäffer, 1847), I. mancipiata (Staudinger, 1871), I. contiguaria (Hübner, 1799), I. aversata (Linné, 1758) e I. deversaria (Herrich-Schäffer, 1847).

Palabras clave: Lepidoptera; Geometridae; Idaea; biología; larvas; quetotaxia; Europa meridional.

\section{ABSTRACT}

G. E. King \& J. L. Viejo Montesinos. 2012. The larvae of the genus /daea Treitschke, 1825 (Insecta, Lepidoptera, Geometridae): New data on its morphology and biology. Grael/sia, 68(2): 249-262 (in Spanish).

The biology and larval chaetotaxy of six species or subspecies were studied in the genus Idaea Treitschke, 1825 through an analysis of the larvae or the larval exuviae of $I$. sericeata calvaria Wehrli, 1927, I. ochrata albida (Zerny, 1936), I. incisaria (Staudinger, 1892), I. bigladiata Herbulot, 1975, I. cervantaria (Millière, 1869) and I. degeneraria (Hübner, 1799), whilst larval biological data was provided on another six species: I. ochrata (Scopoli, 1763), I. macilentaria (Herrich-Schäffer, 1847), I. mancipiata (Staudinger, 1871), I. contiguaria (Hübner, 1799), I. aversata (Linné, 1758) and I. deversaria (Herrich-Schäffer, 1847).

Key words: Lepidoptera; Geometridae; Idaea; biology; larvae; chaetotaxy; southern Europe.

Departamento de Biología (Zoología), Universidad Autónoma de Madrid, C/. Darwin, 2, 28049 Cantoblanco (Madrid), Spain. email: sterrhinae@gmail.com email: joseluis.viejo@uam.es 


\section{Introducción}

Covell (1983) indicó que no se conocen bien los estados inmaduros de los Sterrhinae, por las dificultades en localizar las larvas en estado silvestre, sin embargo, saber que éstas se encuentran a nivel del suelo entre las hojas caídas (Hausmann, 2001), o incluso entre la hojarasca o la arena (Kravchenko et al, 2006; King \& Viejo Montesinos, 2007) en el caso la tribu Sterrhini Meyrick, 1892, ayuda considerablemente a su localización.

Singh (1951) en su trabajo morfológico trató de tres géneros (de distribución europea) de la subfamilia Sterrhinae Meyrick, 1892: Problepsis Lederer, 1853, Scopula Schrank, 1802 e Idaea Treitschke, 1825. Las características de las larvas de Idaea son: cápsula cefálica hipognata, con superfice granular, que se estrecha ligeramente hacia la zona bucal; quetas frontales situadas al mismo nivel que los poros y las quetas de la zona anterolateral de la cápsula cefálica con la siguiente disposición: A2 posterior a A1; $\mathrm{A} 3$ hacia el ocelo 2; L1 casi en línea recta desde el ocelo 1; P1 y P2 en la zona anterolateral de la cápsula, con $\mathrm{P} 2$ por detrás de $\mathrm{P} 1$; ocelo 2 casi equidistante entre los ocelos 1 y 3 y este último más cerca del ocelo 4 que del 2; $\mathrm{O} 2$ que casi toca al ocelo 1; O1 más próxima al ocelo 3 que al 2, siendo a la vez equidistante de los ocelos 3 y 4 ; hilera alargada, roma en el ápice y que se proyecta por debajo de las quetas de los palpos. Abdomen casi cilíndrico, más estrecho en la región torácica y de cutícula granulosa. Las características de las quetas laterales son: en T1, L2 es más alargada que L1; D2 se dispone casi por debajo de D1 y ambas forman una fila vertical con SD2; SD1 por delante de SD2; L3 en posición posterior a SD2 y por detrás de L1. En la región abdominal, SD1 en A1 se sitúa por encima de L2, que se coloca por delante de la queta extra, designada con el término lambda (Singh, 1951), en los uritos A1, A6 y A7, pero más arriba en los uritos A2-A5. En lo que a los espiráculos se refiere, son 'pequeños' de forma 'circular' y con tamaños ligeramente distintos entre A6-A8; el espiráculo de T1 es el más grande.

Otros autores que han contribuido de una manera importante a describir las larvas de Idaea incluyen a Glaser (1958), cuyas descripciones de los estadios L1-L5 se esmeran en sus dibujos de la cápsula cefálica, el labro, los ocelos, las antenas y las mandíbulas, además de las patas torácicas de Idaea longaria (Herrich-Schäffer, 1852); Wiltshire (1962), cuyos detalles de la larva de I. mimetes (Brandt, 1941) hacen mención a la morfología externa de las quetas tanto en L1 como en L5, además de la textura cuticular; también trató de dos especies de Scopula: $S$. adelpharia (Püngeler, 1894) y $S$. minorata (Boisduval, 1833); y Reisser \& Weisert (1977), quienes incluyeron una descripción detallada de las quetas (no su colocación, pero sí su forma) en los estadios L1 y L5 de I. deitanaria (Reisser \& Weisert, 1977).

En la descripción original (Lenz \& Hausmann, 1992) de I. blaesii, cuyas larvas son del tipo 'corto' entre las del género Idaea Hausmann (2004), se incluyó una mención de las quetas en el $1^{\text {er }}$ estadio.

Sannino \& Espinosa $(1999,2002)$ trataron de la morfología y quetotaxia de dos especies europeas: S. turbidaria (Hübner, 1819) e I. seriata canteneraria (Boisduval, 1840) con la terminología de Hinton (1946) y McGuffin (1964); las larvas de $I$. nigra Hausmann \& Bläsius, 2007 son del tipo 'corto' entre las larvas del género Idaea y tienen las quetas en forma de 'tallo' (Hausmann et al, 2007).

Hausmann (2004) presentó la idea de un 'gradiente' de larvas de este género, desde las de tipo 'corto' ('cortas' y 'gruesas' con los 'costados aplanados'), hasta larvas más 'estilizadas' y con menos 'estructura aplanada lateral'.

\section{Material y métodos}

Sabiendo que las larvas del género Idaea se encuentran al nivel del suelo, por debajo de las matas de vegetación o entre la hojarasca (Hausmann, 2001; Kravchenko et al., 2006; King \& Viejo Montesinos, 2007) se iniciaron prospecciones en Madrid (20042012) y en los Alpes Marítimos (Italia) (2008-2011) desde finales de febrero hasta principios de junio. Se inspeccionó la hojarasca, o se rascó el suelo, en sitios abiertos por debajo de las plantas bajas, conservando las larvas halladas en botes bien etiquetados hasta el regreso al laboratorio. Para este estudio no sólo utilizamos las larvas capturadas en el medio natural, sino que también aprovechamos las larvas criadas en cautividad a partir de huevos puestos en el laboratorio, en los fragmentos de la planta nutricia o simplemente en las paredes de los botes (Friedrich, 1976; Soria, 1987; Winter, 2000). La cría de las larvas en cautividad se efectuó en botes de plástico, con las tapas perforadas y el fondo forrado con papel, con los cuidados pertinentes hasta que las larvas efectuaron la metamorfosis (Stehr, 1987a; Winter, 2000). 
Las larvas se guardaron en etanol al 70\% dentro de viales Eppendorf etiquetados con los datos adecuados; por el contrario, las exuvias no se conservaron en alcohol sino en cápsulas por debajo del imago preparado. Las imágenes se tomaron con un estereomicroscopio Leica MZ12.5, acoplado a una cámara de vídeo digital Olympus. Para la fotografía con el MEB el material se montó en stubs con discos adhesivos de carbón, y posteriormente bañados en oro con un metalizador Quorum Q150TS; estas imágenes se tomaron con un equipo Amray 1810 (voltaje acelerador de $10 \mathrm{kV}$ ).

Para la quetotaxia se han seguido los términos de Hinton (1946) (cápsula cefálica; T1, T2) y Dugdale (1961) (A6-A10); la nomenclatura se corresponde con la de Hausmann (2004).

Las muestras de las larvas (y sus imagos correspondientes) que se mencionan en este trabajo se conservan en el Departamento de Biología (Zoología), Universidad Autónoma de Madrid. El material coleccionado en Italia se encuentra depositado en el Museo Regionale de Scienze Naturali, Turín.

\section{ABREVIATURAS}

G.E.K.: Gareth Edward King

L.R.: Luis Romera

co: coxa

escudo to: escudo torácico

esp: espiráculo

mand: mandíbulas

pin: pináculo

A1-A10: se refieren a los uritos de la región abdominal

A1-A3: quetas latero-dorsales en la cápsula cefálica

D1-D2: quetas en la región dorsal

L1-L4: quetas laterales

L1-L5: se refieren al estado larvario entre ecdisis

LR1-LR3: quetas ubicadas en el labro (zona bucal)

O1-O3: se refieren a las quetas ocelares en la cápsula cefálica

PO1-2: quetas antero-dorsales en la cápsula cefálica

SD1-SD2: quetas ubicadas en la zona subdorsal

SO: quetas subocelares en la cápsula cefálica

SV1-SV4: quetas subventrales, su cantidad depende del segmento; son más numerosas en la región abdominal

T1-T3: se refieren a los segmentos de la región torácica

XD1-XD2: quetas ubicadas en el escudo antero-dorsal del segmento $\mathrm{T} 1$

V1: queta en la zona ventral

\section{Resultados}

IDAEA SERICEATA CALVARIA Wehrli, 1927

Larvas halladas en Tres Cantos (Madrid, 727 m, N40 35'49.7; W003 43'11.3): 5.V.07 (5 x L5); 13.IV.08 (5 x L4); 20.IV.08
(3 x L4); 27.IV.08 (1 x L5): Todos los ejemplares a nivel del suelo (arenas de la facies de Madrid: IZCO, 1984) por debajo de matas de Artemisia campestris L. (Asteraceae).

Primer estadio (L1): $1,7 \mathrm{~mm}(\mathrm{n}=2)$ (ex 우 5.VI.10; larvas eclosionadas: 9.VI.10; descripción: 11.VI.10): en conjunto olivácea; rayas corporales ocre rojizas; cápsula cefálica: negruzca. Observación (21.VI.10) $2,1 \mathrm{~mm}(\mathrm{n}=1) ; 3 \mathrm{~mm}(\mathrm{n}=1)$ observación (28.VI.10).

Quinto ESTADiO (L5): 15,8 mm ( $\mathrm{n}=2)$; prepupa: 12,5 mm ( $\mathrm{n}=1)$ (2 ejemplares en alcohol: 13.IV.08); ejemplar: (5.V.07) en conjunto de color ocre; en los flancos, a partir de A4, se destacan dibujos grisáceoblancuzcos que se intensifican en A5; la línea dorsal es blanca y destaca sobre todo en A5-A10; dos puntos negruzcos casi simétricos se colocan a cada lado de esta línea en A3-A6; en la región dorsal se aprecian unos dibujos de diamantes también en A3-A6, el último de estos dibujos es más claro, ocre-blancuzco, y termina en un par de líneas del mismo tono que corren paralelamente a la línea dorsal.

Quetotaxia de L5 $(\mathrm{n}=2)$ : Cápsula cefálica (Figs. 1, 2); hipognata; los ocelos se ubican en una forma casi cuadricular y están bastante distanciados entre sí; ocelos 1 y 5 se colocan a una distancia que equivale casi cuatro ocelos; ocelos 1 y 2 se ubican a dos ocelos el uno del otro; los ocelos 1 y 4 son los más grandes del conjunto, este tamaño equivale a dos veces el ocelo 5 , el más pequeño del grupo (= $75 \%$ del tamaño del resto); ocelos 3 y 4 se encuentran a una distancia el uno del otro que equivale a la mitad de un ocelo, sin tocarse.

Quetas ocelares: O1, O2, O3, esta última se ubica fuera del conjunto de los ocelos; O2 tiene forma de 'mazo' y está colocada al lado del ocelo 1, en cambio, $\mathrm{O} 1$ es más larga y fina y se encuentra al lado del ocelo 3; las quetas A1 y A2 se diferencian entre sí porque $\mathrm{A} 1$ está al mismo nivel que $\mathrm{O} 1$, y también es relativamente larga y fina, mientras que A2 se sitúa al mismo nivel que $\mathrm{O} 1$ y se aprecia la forma parecida a un 'mazo'.

Región torácica $(\mathrm{n}=2)$ : T1: quetas gruesas en su conjunto y en forma de mazo; pináculos de color ocre oscuro; quetas XD1 y XD2 en el escudo cervical; quetas D1 y D2 presentes; L1 y L2 situadas por delante del espiráculo, L1 más larga y más fina; hay quetas SV1 y SV2 (bisetosa); T2-T3: D1 y D2 presentes y dispuestas en pináculos; también destacan las quetas laterales L2, L3 y L1, esta última larga y fina (Fig. 3). 

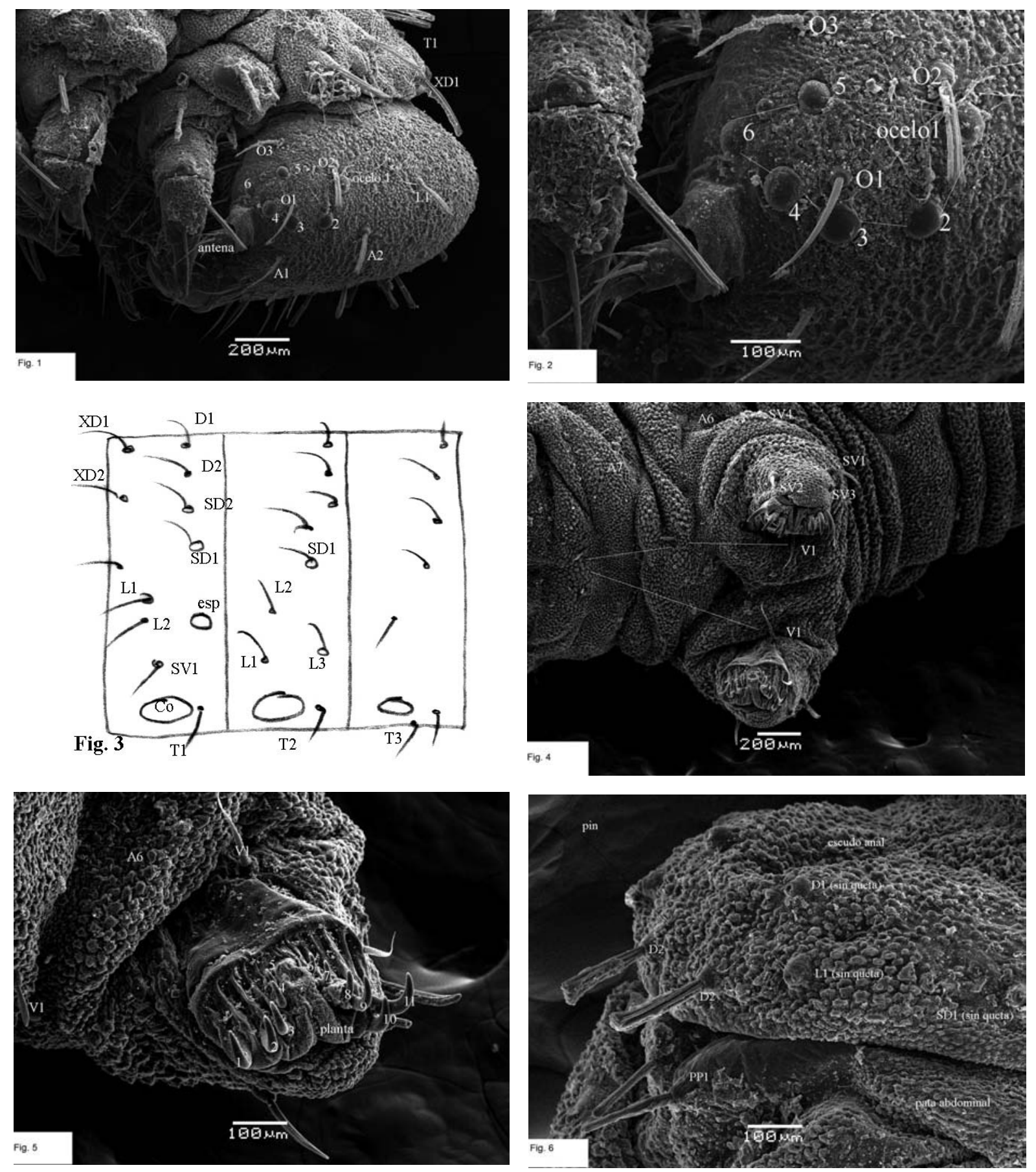

Figs. 1-6.- Idaea sericeata calvaria L5 (13.IV.08, Tres Cantos): 1) cápsula cefálica vista lateral; quetas correspondientes. 2) cápsula cefálica vista lateral; ubicación de los ocelos. 3) región torácica: T1-T3; quetas correspondientes. 4) A6: vista ventral: ganchillos, quetas correspondientes. 5) A6: vista ventral: ganchillos. 6) A10: vista lateral; escudo anal, pata abdominal zona anterior: quetas correspondientes.

Figs. 1-6.- Idaea sericeata calvaria L5 (13.IV.08, Tres Cantos): 1) head capsule lateral view; setae; 2) head capsule lateral view; positioning of stemmata; 3) thoracic region: T1-T3; setae; 4) A6: ventral view; crochets; setae; 5) A6: ventral view; crochets; 6) A10: lateral view; anal shield; anal proleg anterior area; setae. 
Región abdominal (Dugdale, 1961): A6 (Figs. 4, 5) con L4 en la zona superior a la pata abdominal (SV4 según Stehr, 1987b); SV1, SV2 y SV3 en la zona anterior de la pata y V1 en la posterior; 11 ganchillos (Fig. 5) separados (5-planta-6) con los ganchillos 1, 2, 10 y 11 dos veces más largas que los interiores (uniserial, según Dugdale, 1961); A7 (Fig. 6) con SD1, SD2, L1 y L2 que forman una 'cuadrícula' alrededor del espiráculo; A10: escudo anal (Figs. 6) relativamente romo; quetas en aposición: D2 con forma de 'mazo', PP1 más afiladas; zona distal roma.

\section{IDAEA MACILENTARIA (Herrich-Schäffer, 1847)}

1 larva: Tres Cantos (Madrid); 25.IV.09 (1 x L5) a nivel del suelo por debajo de una mata de Artemisia campestris ( $\sigma^{\pi}$ emergió 11.V.09 GEK leg et det.). En cautividad se cría a base de Rumex acetosella L. (hojas, flores).

\section{IDAEA OCHRATA ALBIDA (Zerny, 1936) (Fig. 7)}

Se hallaron 100 larvas a continuación: Tres Cantos: 1.IV.07 (1 x L3), 13.IV.08 (3 x L4), 20.IV.08 (1 x L5), 27.IV.08 (4 x L5), 3.V.08 (4 x L5), 18.V.08 (4 x L5, 1 x L4), 18.IV.09 (12 x L4), 25.IV.09 (5 x L5), 17.V.09 (5 x L5), 24.IV.10 (4 x L4); El Goloso (Madrid: $747 \mathrm{~m}, \mathrm{~N} 40^{\circ} 33^{\prime} 39^{\prime} 3$; W00342'51'9): 1.VI.08 (16 x L5), 14.II.09 (1 x L3), 28.II.09 (1 x L3), 1.III.09 (3 x L4), 29.III.09 (4 x L4), 9.V.09 (1 x L4, 5 x L5), 19.III.10 (1 x L3), 2.IV.10 (1 x L5), 18.IV.10 (7 x L4/L5), 25.IV.10 ( 1 x L5), 8.V.10 (5 x L5), 15.V.10 (3 x L5), 5.III.11 (1 x L4), 12.III.11 ( $1 \mathrm{x}$ L4), 28.III.11 ( $1 \mathrm{x}$ L4), 5.IV.12 ( $1 \mathrm{x}$ L4); todos los ejemplares se encontraron a nivel del suelo por debajo de matas de Artemisia campestris L. (Asteraceae), Rumex crispus L. y $R$. acetosa L. (Polygonaceae), es de destacar la presencia de otras plantas bajas en la proximidad de estas matas: Taraxacum (Asteraceae), Galium (Rubiaceae), Sanguisorba (Rosaceae), Vicia, Ononbrychis, Medicago (Fabaceae), o incluso, Festuca (Poaceae).

Primer ESTADIO (L1) $1,2 \mathrm{~mm}(\mathrm{n}=4)$ (acoplamientos en condiciones de cautividad: huevos obtenidos: 6.VI.10; neonatas: 10.VI.10; descripción: 11.VI.10): conjunto verde oliva pálido; línea dorsal blanca sucia; cápsula cefálica ocre; descripción (21.VI.10): $(n=2) 2 \mathrm{~mm}$ : ventralmente en A2-A5 se destacan máculas negras sobre un fondo blancuzco grisáceo; tanto dorsal como lateralmente transcurren hasta cuatro líneas de color ocre rojizo; cápsula cefálica negra brillante.

CuARTO ESTAdio (L4): $8,9 \mathrm{~mm}(\mathrm{n}=4)$ : ejemplar en ecdisis: conjunto ocre pálido; las patas tanto abdominales como torácicas del mismo tono; cápsula cefálica ocre pálido moteado en ocre oscuro; línea dorsal bordeada en toda su longitud con ocre oscuro, sin embargo, disminuye en intensidad hacia A2; máculas ocre-oscuras repartidas de forma irregular en A2-A8.

Quinto ESTAdio L5 (Fig. 7): 9,2 mm $(\mathrm{n}=17)$ : ejemplar 1 (11,5 mm; 2.IV.10, El Goloso): ocre pálido en conjunto; línea dorsal crema sucia bordeada en ocre oscuro, por esta misma línea transcurre una zona dorsal de color ocre grisáceo; espiráculos ocre dorados; cápsula cefálica ocre pálido moteado en ocre; zonas laterales ocre grisáceo y zonas ventrales ocre moteado; patas torácicas y abdominales de coloración ocre pálido. En la zona dorsal-lateral destacan dos puntos negros asimétricos, tanto en A9 como en A2 y A7. Ejemplar $2(9 \mathrm{~mm}$; en Medicago; 18.IV.10): color de conjunto ocre grisáceo; línea dorsal ocre grisáceo bordeada en ocre oscuro; paralelamente transcurre una zona ocre grisáceo y luego dos líneas dorsales también ocre grisáceas; cápsula cefálica y patas torácicas y abdominales también ocre pálido; A4-A7 con máculas ocre-oscuras situadas inmediatamente por debajo de los espiráculos, que son asimismo de color ocre y se ubican dentro de las rugosidades laterales aplanadas que caracterizan la especie; ventralmente se distinguen unos dibujos irregulares de tonalidades ocre oscuro; quetas doradas. Ejemplar 3 (11 mm; en Vicia; 18.IV.10): línea dorsal ocre pálido en T1-T3; en A1-A10 las márgenes de la línea son de tono ocre e intensidad variable; A8-A10 más oscuros; a lo largo del resto de la línea el color apenas resulta visible; destaca la presencia de conspicuas máculas ocres en A2, A3, A8.

Quetotaxia L5: cápsula cefálica (Figs. 8, 9) $(n=3)$ hipognata; los ocelos en su conjunto forman un 'semicírculo'(ocelo 6 se queda al margen); ocelo 1 es $50 \%$ más amplio que los demás ocelos; los ocelos 1 a 3 se encuentran a una distancia uno del otro que equivale al ancho de un ocelo y un $25 \%$ más; el ocelo 4 se coloca a un ocelo del 3; y los ocelos 5 y 6 se ubican a dos ocelos de distancia de los demás; los ocelos 1 y 5 se encuentran separados entre sí a una distancia aproximada de cinco ocelos (Fig. 9); O2 se ubica al lado del ocelo 1, O1 al lado del ocelo 3, casi tocando la base de éste, el ocelo 5 se sitúa alejado del conjunto y O3 al lado del ocelo 6; el ocelo 2 adopta forma de 'mazo', mientras $\mathrm{O} 3$ es más fina distalmente y $\mathrm{A} 3$ se parece a $\mathrm{O} 2$. En la Fig. 10, correspondiente a la zona bucal, se indican las quetas ubicadas en el labro, que son relativamente cortas: LR 1 mide $2 / 3$ de la 


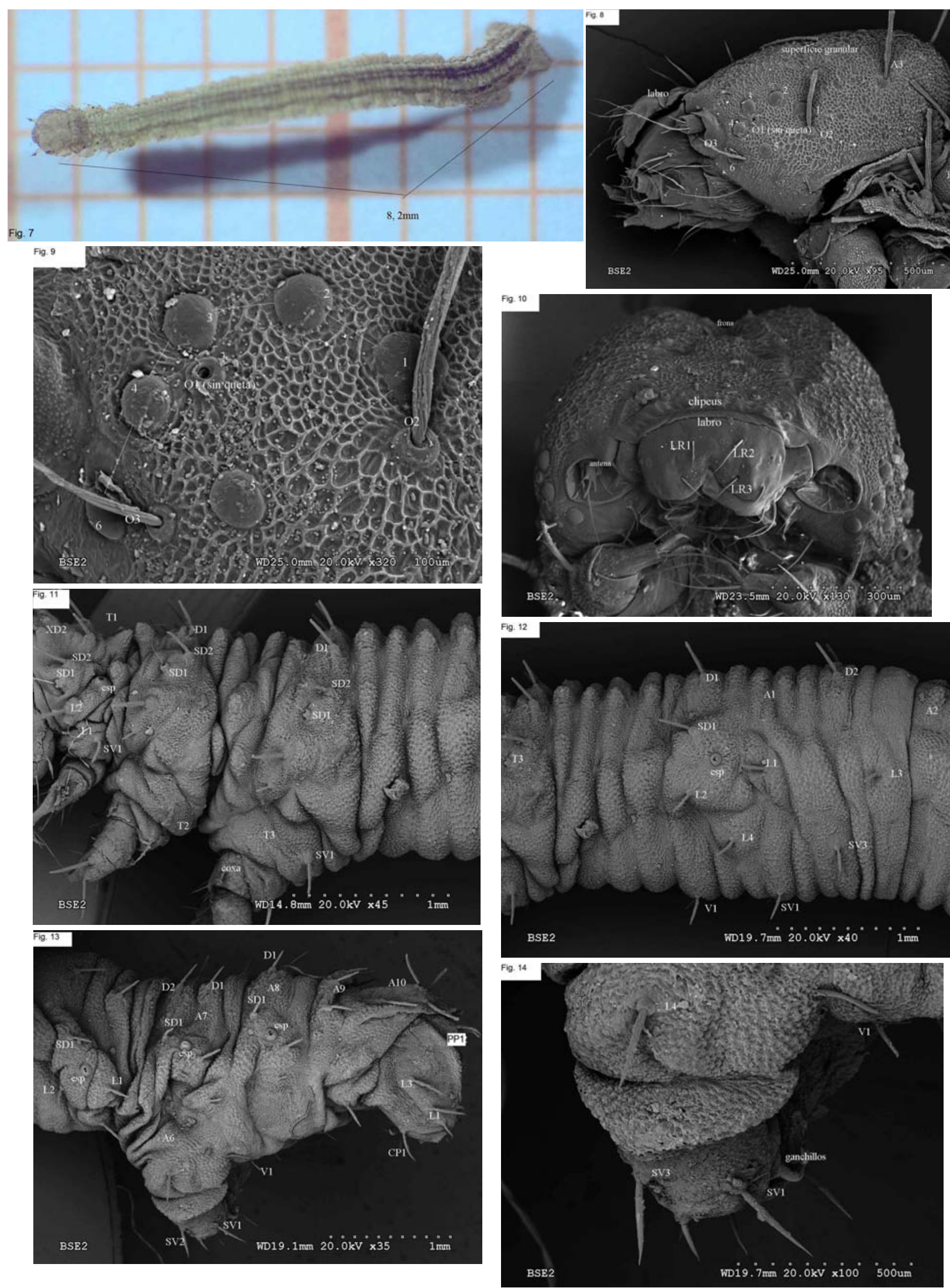

Graellsia, 68(2), Diciembre 2012, pp. 249-262 - ISSN: 0367-5041 doi:10.3989/graellsia.2012.v68.054 

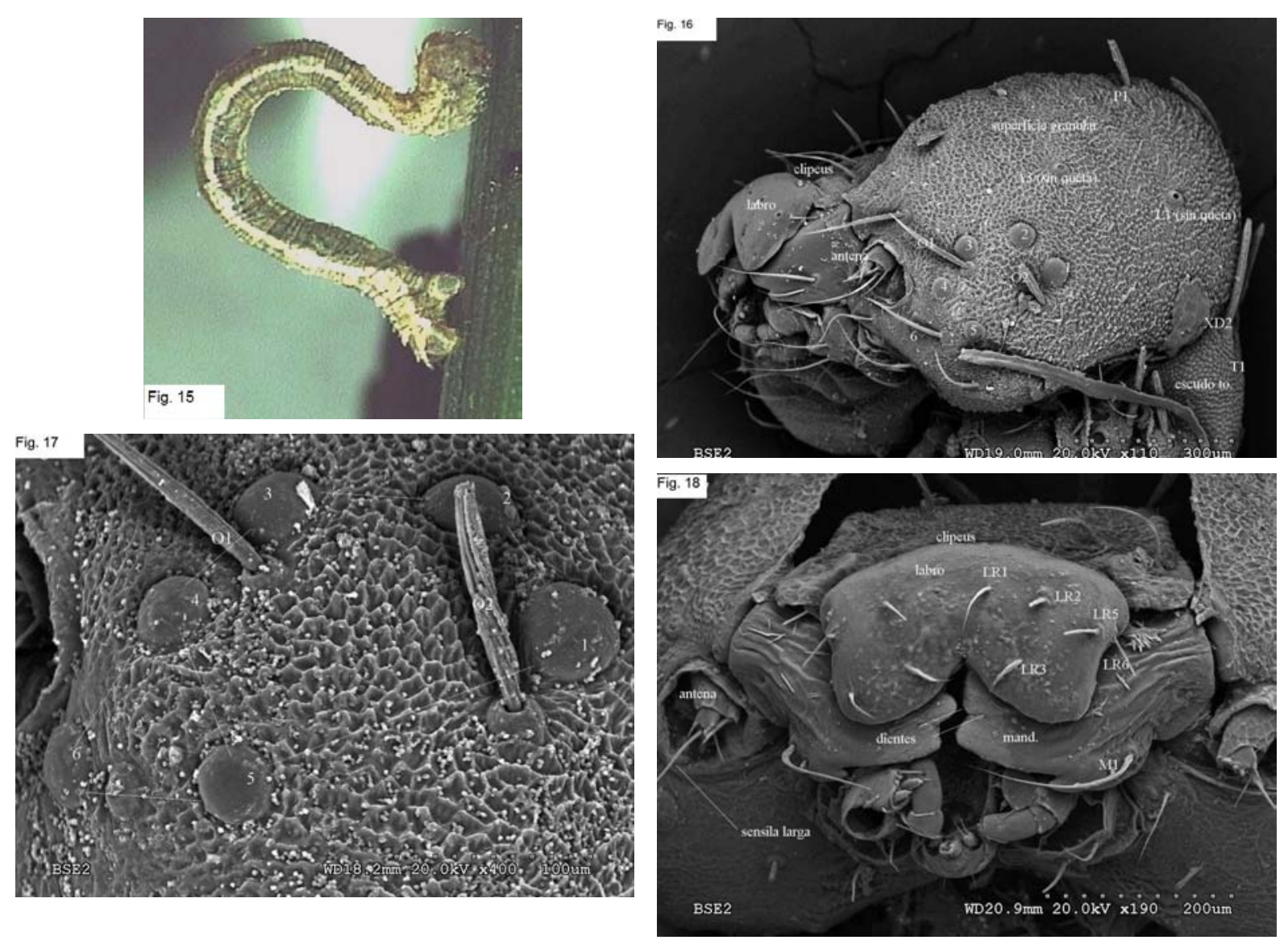

Figs. 15-18.- Idaea incisaria L5. 15) ex 오, Ciempozuelos, 7.X.07. 16) cápsula cefálica (ex 우 22.V.02, Cantoblanco) vista lateral: quetas correspondientes. 17) cápsula cefálica: ubicación de los ocelos vista ventral; distancia entre los ocelos. 18) labro vista dorsal; quetas correspondientes.

Figs. 15-18.- Idaea incisaria L5. 15) larva ex 우, Ciempozuelos, 7.X.07. 16) head capsule lateral view (ex 우 22.V.02, Cantoblanco); stemmata; setae. 17) head capsule ventral view: stemmata; setae. 18) mouthparts: dorsal view; labrum; setae.

longitud de LR2 y LR3 (Hinton, 1946; Ahola \& Silvonen, 2005).

Región torácica $(\mathrm{n}=3)$ : T1: quetas con forma de 'mazos', pináculos ocres y destacables; SD1 se dispone en un pináculo más ancho (Markl \& Tautz, 1975) y SD2, SD1, L2 y L1 en orden descendente anterior al espiráculo; L1 muy fino y largo ubicado en un pináculo (Fig. 11).

Región abdominal: A1 (Fig. 12): según Dugdale (1961), porta cuatro quetas laterales: L1, L2, L3, L4; alrededor del espiráculo se disponen SD1, L1 y L2 formando un triángulo y L3 se encuentra en

Figs. 7-14.- Idaea ochrata albida L5. 23.IV.11, El Goloso: 7) aspecto de la larva. 8) L5 cápsula cefálica: quetas en la proximidad de los ocelos; vista lateral (exuvium: larva: V.10. El Goloso); 9) quetas en la proximidad de los ocelos. 10) cápsula cefálica: vista dorsal zona bucal (IV.09, El Goloso). 11) (23.IV.11, El Goloso); región torácica vista lateral; quetas correspondientes. 12) región abdominal $A 1$ vista lateral; quetas correspondientes. 13) región abdominal A6-A10 vista lateral; quetas correspondientes. 14) A6 pata abdominal vista anterior; quetas correspondientes.

Figs. 7-14.- Idaea ochrata albida L5. 23.IV.11, El Goloso: 7) larva. 8) head capsule: lateral view; stemmata, setae. 9) stemmata; setae. 10) mouthparts: labrum; setae. 11) thoracic region: lateral view; setae. 12) abdominal region: A1 lateral view; setae. 13) A6-A10 lateral view; setae. 14) A6: proleg; setae. 
línea casi recta al mismo nivel que L1; L4 y SV3 se sitúan al mismo nivel pero bien separadas entre sí; L4 forma un triángulo con V1 y SV1 está en la zona ventral; A6 (Figs. 13, 14): L4 se ubica en la zona superior a la pata (Dugdale, 1961) y hay seis subventrales (SV1, SV3) en la zona anterior de la pata; alrededor del espiráculo se encuentran SD1, L1 y L2 que forman un triángulo, con L1 y L2 al mismo nivel; A10: escudo anal de forma triangular; D2 en la zona apical están en aposición con las quetas PP1 en la zona anterior de las patas anales que se doblan distalmente (Fig. 13).

\section{IDAEA OCHRATA (Scopoli, 1763)}

Larvas (3) 27, 28.V.11 (3 x L5): Sant'Ana di Valdieri (1166 m; N44ำ1'10.4; E007 18'02.1; prov. Cuneo, ITALIA) (emergieron: VI. 11: $20^{\top} 1$ 우 (gen. prep. 3742) GEK leg et det)] halladas por debajo de las matas de Rumex conglomeratus Murray (Polygonaceae).

\section{IDAEA INCISARIA (Staudinger, 1892) (Fig. 15)}

Larva (1): Ciempozuelos (Madrid: 518 m, N4009’09.1; W00336'27.1) a nivel del suelo por debajo de Antirrhinum majus L. (Scrophulariaceae) (King \& Viejo Montesinos, 2010).

QuetotaXia: cápsula cefálica: (L5; exuvias $\mathrm{n}=2$ ) (Figs. 16, 17): superficie granular; los ocelos en su conjunto forman un semicírculo (sin contar el ocelo 6); el ocelo 1 es el más amplio, 25\% más que el ocelo 2; O2 (con forma de 'mazo') se ubica al lado del ocelo 1 y O1 (más 'estilizada' que $\mathrm{O} 2$ y muy fina distalmente), al lado del ocelo 3; estas dos quetas casi tocan la base de sus ocelos respectivos; en lo que se refiere a la ubicación de los ocelos, los ocelos 5 y 6 se alejan del conjunto ubicándose a una distancia de dos veces su tamaño del conjunto de los ocelos 1-4; en cambio, los ocelos 1-3 se colocan a una distancia equivalente al tamaño de cada uno en este conjunto de tres; los ocelos 3 y 4 son los más cercanos al uno al otro con queta $\mathrm{O} 2$ casi equidistante de los dos, pero más cerca del ocelo 3; labro con su complemento de quetas de la misma longitud; LR6 un poco más larga (Fig. 18) (Ahola \& Silvonen, 2005).

\section{IDAEA MANCIPIATA (Staudinger, 1871)}

Larva (1): Tres Cantos; 17.V.09 (1 x L5) a nivel del suelo por debajo de Rumex acetosa (pupó 23.V.09; $\sigma^{7}$ emergió 3.VI.09; gen. prep. 3486 GEK leg et det.). Se cría a base de hojas marchitas de Rumex en condiciones de cautividad.

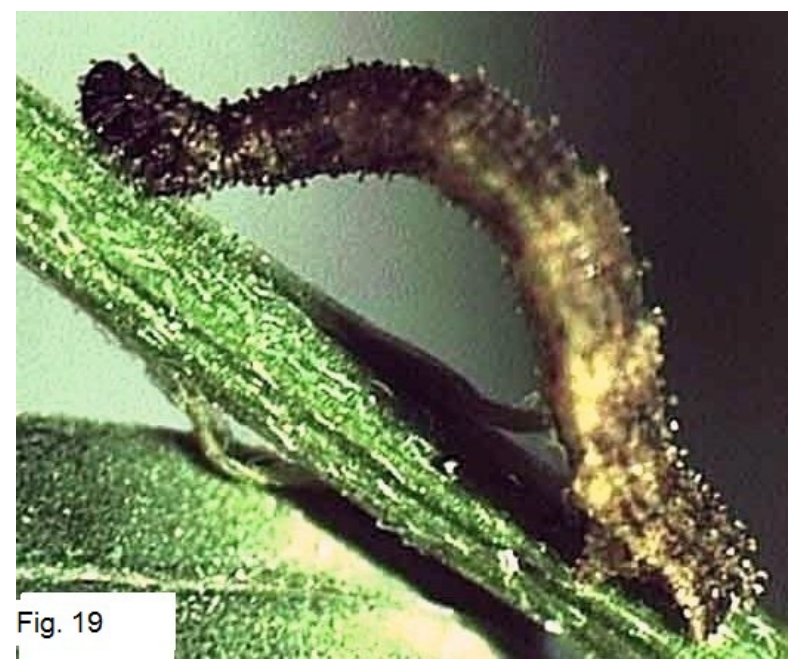

Fig. 19.- I. bigladiata: L4 (ex ㅇ 26.V.07; foto: 23.XI.07), Tres Cantos; en hojas marchitas de Medicago.

Fig. 19.- I. bigladiata: L4 larva (ex ㅇ 26.V.07; photo: 23.XI.07), Tres Cantos; reared on withered leaves of Medicago.

\section{IDAEA BIGLADIATA Herbulot, 1975 (Fig. 19)}

Larvas (2): Tres Cantos: a nivel del suelo por debajo de una mata de Artemisia campestris (King \& Viejo Montesinos, 2007).

QuetotaXia: L3 (ex $0^{\top}$ V. 06, Canillejas, t. m. de Madrid; 4,5 mm; ejemplar en alcohol) $(\mathrm{n}=1)$ : por lo general las quetas se dividen en dos tipos: en forma de 'abanico' (D1, D2 desde T3 hasta A8, también V1 desde T3 hasta A6) y en forma de 'mazo' (D1, D2, XD1, XD2 -escudo torácico- en T1; también A10 en el escudo anal y SD1, D1, D2); pata anal: PP1; pináculos de color ocre oscuro.

Región torácica: T1: SD1 largo y fino. Región abdominal: A1: SD1, L2 y L1 forman un 'triángulo' alrededor el espiráculo y L4 y L3 se disponen al mismo nivel pero bien separadas; A6: SD1, L2 y L1 forman un triángulo alrededor el espiráculo; SV1, SV2 y SV3 en la zona anterior de la pata abdominal; A7: L2 y L1 al mismo nivel por debajo del espiráculo; A8: L1, L2 y L3 se ubican por debajo del espiráculo con L3 en la zona posterior del urito.

\section{IDAEA CERVANTARIA (Millière, 1869) (Fig. 20)}

Larvas (3): Ciempozuelos: a nivel del suelo por debajo de Gypsophila struthium subsp. struthium L. in Loefl. (Caryophyllaceae) (King \& Viejo Montesinos, 2007, 2010); 31.V.08: (2 x L2) en flores de Helianthemum squamatum (L) 

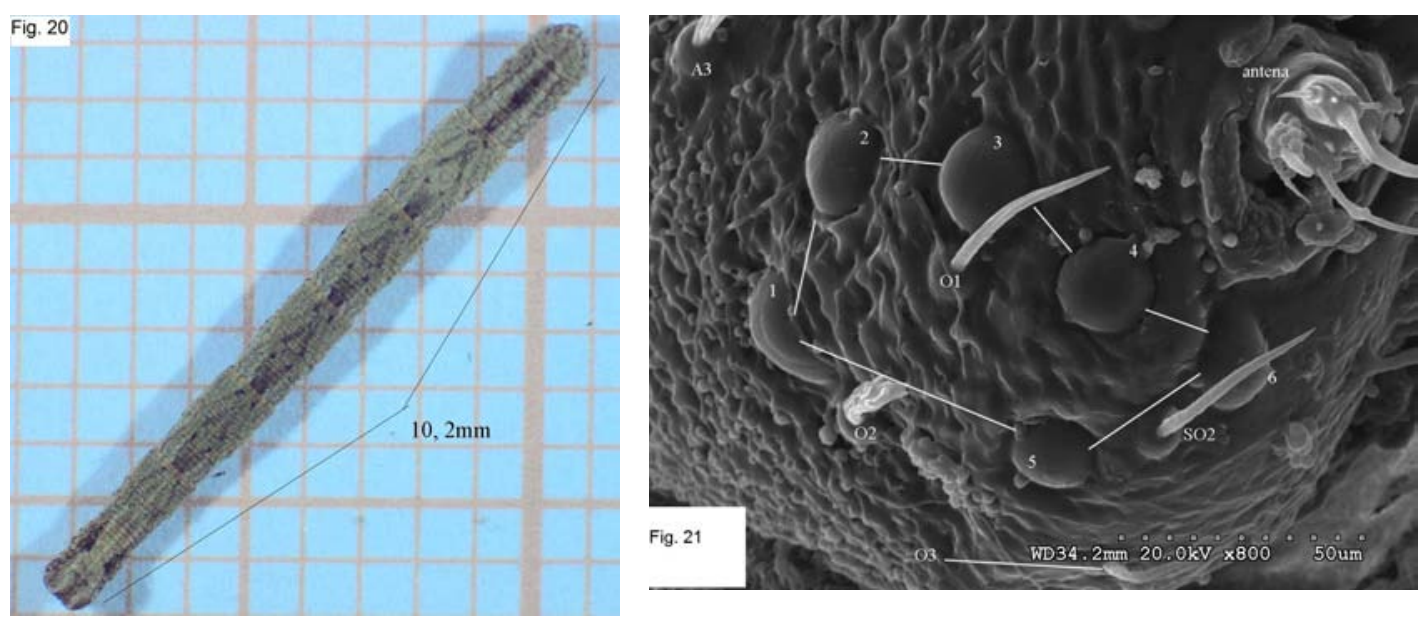

Figs. 20-22.- Idaea cervantaria L5 exuvium (19.III.11, Ciempozuelos). 20) Aspecto del ejemplar. 21) cápsula cefálica vista lateral; quetas correspondientes. 22) cápsula cefálica vista dorsal: quetas.

Figs. 20-22.- Idaea cervantaria L5 exuvium (19.III.11, Ciempozuelos). 20) larva L5. 21) head capsule lateral view; setae. 22) head capsule; dorsal view; setae.

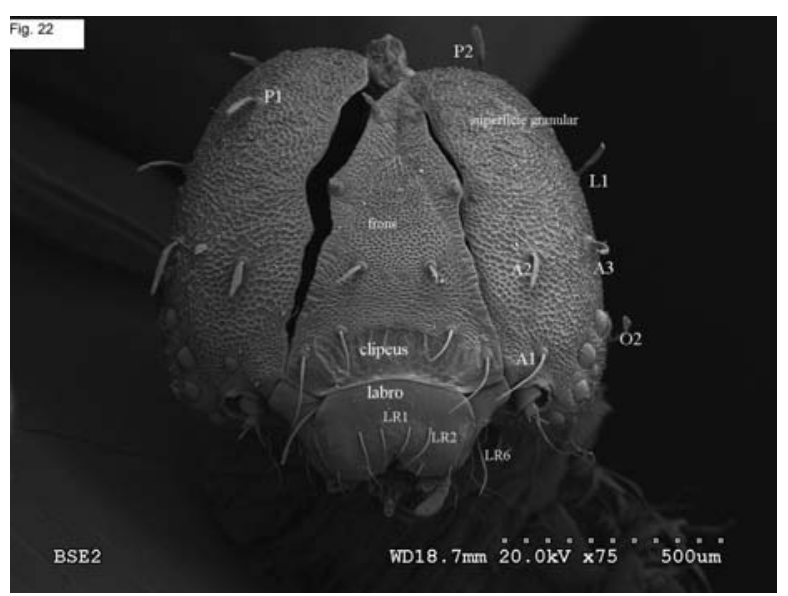

Dum. Coulb. (Cistaceae); 19.III.11 (1 x L5) por debajo de Antirrhinum majus (Fig. 20).

Quinto ESTADIO (L5): 10,2 mm $(\mathrm{n}=1)$ larva de morfología variable, en A2 destacan manchas ocres de forma rectangular que se reparten lateralmente $\mathrm{y}$ disminuyen en A1; A4 de color paja dorsalmente y grisáceo azulado con trazos ocres en la región ventral; A6 y A10 más pálidos con línea dorsal ocre ligeramente bifurcada hacia A5 mientras que en A9 la línea se ve más oscura; patas abdominales de color blanco sucio, pináculos ocres; trazos ocres en segmentos alternativos; patas torácicas translúcidas; pináculos ocres y quetas en T1-T3; cápsula cefálica de tonalidad pajiza pálida reticulada lateralmente; ocelos negros, antenas de color ámbar.

QUETOTAXIA: L1: 2,3 mm ( $\mathrm{n}=2)(e x$ ㅇ 7.V.05); cápsula cefálica hipognata (Figs. 21, 22); quetas por lo general en forma de 'mazo', A1 más larga y fina; los ocelos casi completan un círculo, con la excepción del ocelo 6; ocelos 2-6 ubicados a una distancia de cada uno que equivale a un ocelo; ocelo 1 separado del 2 por una distancia que equivale a dos ocelos; ocelo 6 del ocelo 5 , solo por un ocelo; ocelo 5 del 1, por cuatro ocelos. $\mathrm{O} 2$ situada al lado de ocelo 1; O1 al lado del ocelo 3 y O3 más caudal. SO2 se localiza entre los ocelos 5 y 6 (Fig. 21); A1-A3 forman un 'triángulo': A2 y A3 se colocan a la misma altura dorso-lateralmente, A1 se encuentra a la misma altura que el ocelo 3; L1 se ubica por encima de A3, siendo más caudal en la zona lateral de la cápsula cefálica; y P2 se dispone en el ápice de la misma. Labro: LR1 y LR2 muy finas; LR6 casi dos veces más larga (exuvium L5: 19.III.11) [Fig. 22)] (Ahola \& Silvonen, 2005).

Región torácica $(\mathrm{n}=2)$ : L1: T1 (Fig. 23): quetas en forma de 'mazo'; SD1 (más larga y fina), L1 y L2 se encuentran alrededor del espiráculo en forma de 'triángulo de dimensiones reducidas'; SV1 y SV2 en la zona posterior; T2: SD1 en la porción caudal del espiráculo; L1, L2 y L3 en línea descendente; SV1. 

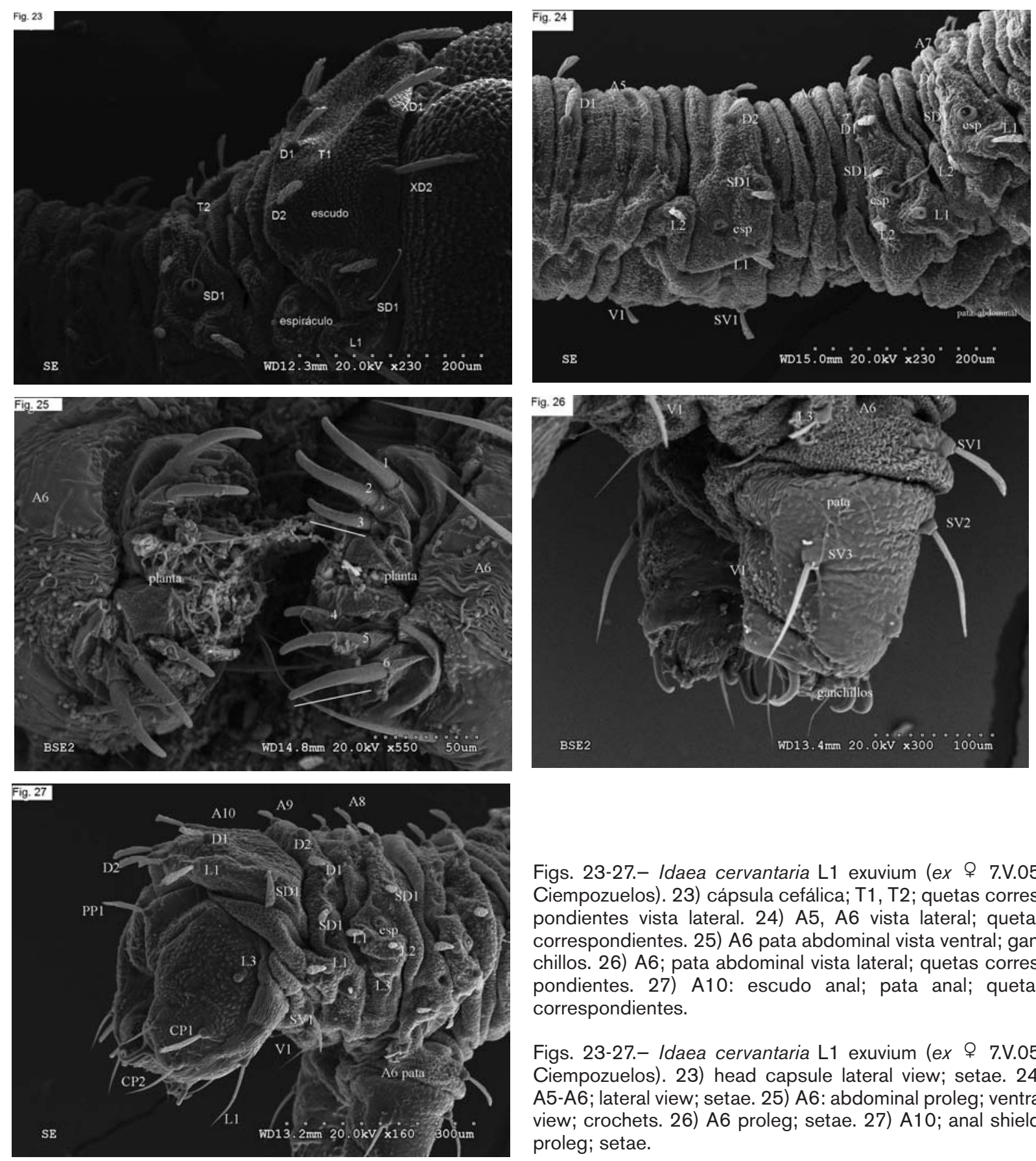

Figs. 23-27.- Idaea cervantaria L1 exuvium (ex 우 7.V.05, Ciempozuelos). 23) cápsula cefálica; T1, T2; quetas correspondientes vista lateral. 24) A5, A6 vista lateral; quetas correspondientes. 25) A6 pata abdominal vista ventral; ganchillos. 26) A6; pata abdominal vista lateral; quetas correspondientes. 27) A10: escudo anal; pata anal; quetas correspondientes.

Figs. 23-27.- Idaea cervantaria L1 exuvium (ex $९$ 7.V.05, Ciempozuelos). 23) head capsule lateral view; setae. 24) A5-A6; lateral view; setae. 25) A6: abdominal proleg; ventral view; crochets. 26) A6 proleg; setae. 27) A10; anal shield, proleg; setae.

Región abdominal (Fig. 24): A5: SD1, L1 y L2 forman un 'triángulo' alrededor del espiráculo; SV1. Pata abdominal A6 (Figs. 25, 26): SD1, L1, L2 y, luego, L3, y en la zona anterior de la pata: SV1, SV2 y SV3; 6 ganchillos separados por una planta, los exteriores son más largos (Fig. 26) (se describieron como mesoseries no continuas Dugdale (1961)]; A8 (Hinton, 1946): SD1 más alejada del espiráculo; L1 y L2 al mismo nivel por debajo del espiráculo, L3 en línea descendente; A9: D2, D1, SD1, L1 y L2 colocados en línea descendente; A10: escudo anal más o menos romo; quetas 
en forma de 'mazo'; en cambio, en la zona posterior de la pata CP2, L1 son relativamente 'largas' y 'finas' (Fig. 27) (Dugdale, 1961).

\section{IDAEA CONTIGUARIA (Hübner, 1799)}

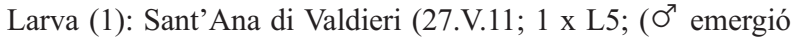
VI. 11; gen. prep. 3740 GEK leg et det.)] a nivel del suelo por debajo de una mata reducida de Silene sp. (Caryophyllaceae) entre matas más importantes de Rumex conglomeratus Murray (Polygonaceae). En cautividad se cría a base de R. acetosella L.

\section{IDAEA AVERSATA (Linné, 1758)}

Larva (1): Sant'Ana di Valdieri; (28.V.11; 1 x L5) ( ㅇ emergió VI. 11; gen. prep. 3739 GEK leg et det.) ] a nivel del suelo por debajo de Rumex conglomeratus . En cautividad se cría a base de $R$. acetosella L.

\section{IDAEA DEGENERARIA (Hübner, 1799) (Fig. 28)}

Larva (1): El Goloso; 27.II.11 (1 x L5) a nivel del suelo por debajo de Sanguisorba officinalis L. (Rosaceae) En cautividad se cría a base de $R$. acetosa (pupó 25.III.11; pupa: 30.III.11 $\sigma^{7}$ emergió 12.IV.11; GEK leg et det.).

QuetotaXia: L5 (exuvium): $16 \mathrm{~mm}(\mathrm{n}=1$ : $\mathrm{El}$ Goloso); las quetas por lo general se alargan distalmente; cápsula cefálica hipognata (Figs. 29, 30); los ocelos en su conjunto forman casi un 'rectángulo' (sin incluir al ocelo 6 en dicho conjunto); ocelos 2-6 del mismo tamaño; el ocelo 1 es ligeramente mayor (un 10\%), mientras que el ocelo 5 es un $10 \%$ menor; ocelos 2-4 separados entre sí por una distancia semejante al $75 \%$ del tamaño de un ocelo; en cambio, el ocelo 1 se coloca a dos ocelos del ocelo 2 y a tres ocelos del ocelo 5 , el cual a su vez se encuentra a dos ocelos del ocelo 4 y a tres del ocelo 6; queta O2 (la más 'gruesa'de las quetas ocelares) se ubica al lado del ocelo 2 sin que toque su base; $\mathrm{O} 1$ se encuentra equidistante entre los ocelos 3 y 4 ; $\mathrm{SO} 2$ se sitúa entre los ocelos 5 y 6; O3 se encuentra a tres ocelos del ocelo 5 al mismo nivel que éste; A1 se ubica por delante de los ocelos y al mismo nivel que $\mathrm{O} 1$; junto con $\mathrm{A} 2$ y $\mathrm{A} 3$ se forman un triángulo, con A1 la más larga y fina del conjunto. Región torácica: T1 (Fig. 31): las quetas tienen forma de 'espada', con la excepción de SD2 que es más gruesa, sobre todo en comparación con SD1, tres veces más larga que ésta y más fina, y además se halla ubicada en el pináculo en forma de 'domo'; A10 (Fig. 32): a diferencia de las quetas en T1, tienen forma de 'mazas' las que se encuentran en el escudo anal; las quetas ubicadas en la pata anal se alargan distalmente (información no disponible del resto de los uritos).

\section{IDAEA DEVERSARIA (Herrich-Schäffer, 1847)}

Larva (1): Vallone de Chistofort (Sant'Ana di Valdieri, 1291m; N44 11'37.9; E007 21'27.6; CUNEO, ITALIA; 29.V.11 (1 x L5) ( + emergió VI. 11; gen. prep. 3741 GEK leg et det.) a nivel del suelo por debajo de Rumex conglomeratus. En cautividad se cría a base de $R$. acetosella L.

\section{Discusión y conclusiones}

En el presente trabajo se ha analizado la quetotaxia de seis taxones en el género Idaea: Idaea sericeata calvaria (L5), I. ochrata albida (L5), I. incisaria (L5; cápsula cefálica), I. bigladiata (L3), I. cervantaria (L1) e I. degeneraria (L5). Se trata de datos inéditos pues únicamente $I$. sericeata calvaria se consideraba relativamente bien analizada: Chrétien (1899) describió la morfología de la larva (L1-L5), mientras que Gómez de Aizpúrua et al (2011) ofrecieron imágenes de la larva en el último estadio. No se ha estudiado la subespecie ibérica, $I$. ochrata albida, sino ejemplares daneses de la subespecie nominotípica centroeuropea (Kristensen, 1966). Se han ofrecido datos morfológicos inéditos de los estadios larvarios de estos taxones: I. sericeata calvaria (L1, L5), I. ochrata albida (L1, L4, L5) e I. cervantaria (L5).

Singh (1951), Wiltshire (1962), Reisser \& Weisert (1977), Lenz \& Hausmann (1992) y Sannino \& Espinosa (2002) han hecho hincapié en las quetas con forma de 'mazo' (espatoliforme, según Sannino \& Espinosa, 2002) entre las larvas de Idaea; en nuestro estudio de seis taxones se ha apreciado este característica sinapomórfica de las quetas en forma de 'mazo' (p. ej. I. sericeata calvaria), pero, en cambio, estas estructuras en I. bigladiata presentan forma de 'mazo' y de 'abanico', mientras que en las larvas de I. degeneraria la forma correspondiente resulta ser más 'afilada'. En los seis taxa se ha apreciado la diferencia en forma entre las quetas $\mathrm{O} 1, \mathrm{O} 2$ y $\mathrm{O} 3$. $\mathrm{La}$ queta $\mathrm{O} 3$, por ejemplo, se estrecha distalmente en comparación con O2. Según Hassenfuss (1963), la longitud y grosor de las quetas en la zona de los stemata se relacionan con las estrategias de las larvas en cuanto al ámbito ocupado se refiere, probablemente, entre las Idaea se asocia con el hecho de que viven a ras del suelo. 

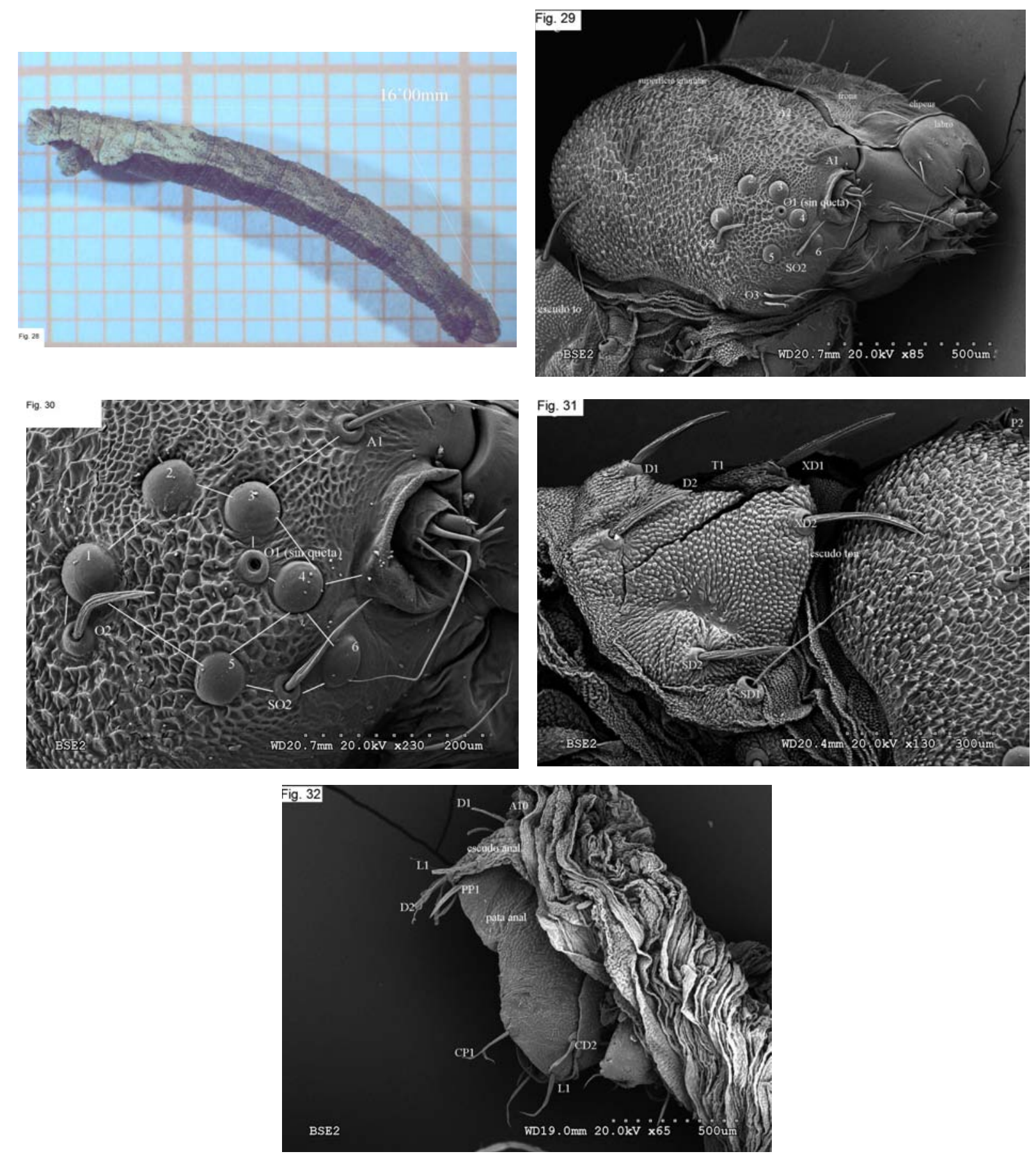

Figs. 28-32.- Idaea degeneraria L5 . 28) Ejemplar capturado el 27.Il.11 en El Goloso. 29) Cápsula cefálica vista lateral; quetas correspondientes. 30) Cápsula cefálica; ubicación de los ocelos vista ventral; distancia entre los ocelos; quetas correspondientes. 31) T1; región torácica; quetas correspondientes. 32) A10; escudo anal; pata anal; quetas correspondientes.

Figs. 28-32.- Idaea degeneraria L5. 28) Specimen from El Goloso 27.II.11. 29) Head capsule; lateral view; setae; mouthparts. 30) Head capsule; ventral view; stemmata; setae. 31) thoracic region; T1; setae. 32) abdominal proleg; A10; anal shield; setae. 
Entre los doce taxones analizados en el presente trabajo, de seis se han aportado datos biológicos nuevos que se indican a continuación: de $I$. mancipiata se han ofrecido datos biológicos inéditos de esta especie con el hallazgo de una larva; los datos ex larvas de $I$. contiguaria, I. aversata, I. degeneraria e $I$. deversaria han incrementando la información disponible en Ebert \& Steiner (2001); en el caso de I. macilentaria, ya se conocía una breve descripción de la morfología, además de la biología en cautividad de la larva (Gómez de Aizpúrua, 1987; Ebert \& Steiner, 2001).

Las larvas capturadas con motivo del estudio actual se encontraron a nivel del suelo, tal vez enroscadas, por debajo de las matas de las plantas, por ejemplo Artemisia campestre, que no se incluye como planta nutricia, sin embargo, otras plantas bajas, por ejemplo Rumex o Vicia, sí forman parte de la gama alimenticia de las larvas, al igual que la hojarasca que se reconoce como fuente alimenticia para las larvas del género Idaea (Chrétien, 1899; Covell, 1983; Ebert \& Steiner, 2001). El hecho de que las larvas se comporten como detritívoras se interpreta como una adaptación a los rigores del clima mediterráneo en donde este género se diversifica mucho (Hausmann, 2004).

\section{Agradecimientos}

A la Sección de Investigación de la Universidad Autónoma de Madrid para la concesión de una beca en septiembre de 2011 (Proyecto 541D708 y 541D731, nov. 2011-enero 2012) que ofreció la posibilidad de colaborar con David Wagner afincado en la Universidad de Connecticut, EEUU, en cuyas instalaciones se redactó este trabajo. A la plantilla en el SIDI de la Universidad Autónoma de Madrid sin cuya ayuda a lo largo del verano del 2011 hubiera sido imposible obtener las imágenes de las larvas con el MEB, en el mismo sentido a la Universidad de Concepción, Chile en cuyas instalaciones se consiguieron las imágenes iniciales en el MEB. Al European Distributed Institute of Taxonomy (EDIT) (Stuttgart, Berlín), y luego, a la plantilla del Parco Nacionale delle Alpi Marittime, Valdieri, Italia, cuyos fondos (dentro del proyecto: Alcotra 2007-2013 y su programa: Generalized Biological Inventory) subvencionaron los viajes y el alojamiento, entre agosto del 2008 y septiembre del 2011. A los revisores del manuscrito, sobre todo a Enrique García Barros, por sus comentarios útiles.

\section{Referencias}

Ahola, M. \& Silvonen, K., 2005. Larvae of Northern European Noctuidae, Tomo I. Kuva Seppälä Yhtiöt Oy. Helsinki. 657 pp.
Chrétien, P., 1899. Les premiers états de l'Acidalia sericeata $\mathrm{Hb}$. (Lép.). Bulletin de la Société entomologique de France, 1899(7): 127-129.

Covell, C. V., 1983. The state of our knowledge of the Neotropical Sterrhinae (Geometridae). Second Symposium on Neotropical Lepidoptera, Arequipa: 1: 1-23.

Dugdale, J. S., 1961. Larval characters of taxonomic significance of New Zealand Ennomines (Lepidoptera: Geometridae). Transactions of the Royal Society of New Zealand, 16: 215-233.

Ebert, G. \& Steiner, A., 2001. Sterrhinae. In: Die Schmetterlinge Baden-Wüttermbergs, Band 8: Nachfalter VI, Geometridae. Verlag E. Ulmer. Stuttgart. 541 pp.

Friedrich, E., 1976. Handbuch der Schmetterlingszucht, Europäische Arten. Franck'sche Verlagshandlung und Kosmos-Verlag. Stuttgart. 186 pp.

Glaser, W., 1958. Beschreibung der ersten Stände Acidalia longaria HS (Lepidoptera: Geometridae). Entomologisches Nachrichtenblatt Österreichischer und Schweizer Entomologen, 10(2): 29-31.

Gómez de Aizpúrua, C., 1987. Biología y morfología de las orugas, Tomo III, Geometridae. Boletín de Sanidad Vegetal, 8(15): 1-238.

Gómez de Aizpúrua, C., González Granados, J. \& Viejo Montesinos, J. L., 2011. Mariposas y sus biotopos, Reserva Natural El Regajal-mar de Ontígola, Lepidoptera V memoria 2009-10. Comunidad de Madrid. $310 \mathrm{pp}$.

Hassenfuss, I., 1963. Eine vergleichend-morfologische Analyse der regulären Borstenmuster der Lepidopterenlarven. Zeitschrift für Morphologie und Ökologie der Tiere, 52: 197-364.

Hausmann, A., 2001. Introduction. Archiearinae, Orthostixinae, Desmobathrinae, Alsophilinae, Geometrinae. In: A. Hausmann (ed.). The Geometrid Moths of Europe, vol. 1. Apollo Books. Stenstrup: 1-282.

Hausmann, A., 2004. Sterrhinae. In: A. Hausmann (ed.). The Geometrid Moths of Europe, vol. 2. Apollo Books. Stenstrup: 1-600.

Hausmann A., Miller, M. A., Leipnitz, M. \& Bläsius, R., 2007. Idaea nigra Hausmann \& Bläsius, sp. $n$. from La Gomera, Canary Islands, Spain (Lepidoptera; Geometridae, Sterrhinae). SHILAP Revista de Lepidopterología, 35(140): 499-505.

Hinton, H. E., 1946. On the homology and nomenclature of the setae of lepidopterous larvae, with some notes on the phylogeny of the Lepidoptera. Transactions of the Entomological Society of London, 97: 1-37.

Izco, J., 1984. Madrid Verde. Ministerio de Agricultura, Pesca y Alimentación. Madrid. 517 pp.

King, G. E. \& Romera, L., 2004. Contribución al estudio de la biología de los geométridos en Madrid (España), con datos nuevos sobre sus plantas nutricias (Lepidoptera: Geometridae). SHILAP Revista de Lepidopterología, 32(126): 139-147. 
King, G. E. \& Viejo Montesinos, J. L., 2007. Comentarios y aportaciones nuevas de especies en el género Idaea Treitschke, 1825 y Scopula Schrank, 1802 en Madrid (Lepidoptera: Geometridae; Sterrhinae). Boletín de la Asociación Española de Entomología, 31(3.4): 167186.

King, G. E. \& Viejo Montesinos, J. L., 2010. Geometrid larvae and their food-plants in the south of Madrid (Central Spain) (Geometridae). Nota lepidopterologica, 33(1): 4-61.

Kravchenko, V. D., Hausmann, A. \& Miller, G. C., 2006. Deserticolous Noctuidae from Israel: New host-plant records and descriptions of larval habitats,(Lepidoptera: Noctuidae). Mitteilungen der Münchner Entomologischen Gesellschaft, 96: 29-42.

Kristensen, N. P., 1966. Notes on Sterrha ochrata, a moth new to the Danish fauna (Lepidoptera: Geometridae). Entomologiske Meddelelser, 34: 214220.

Lenz, J. \& Hausmann, A., 1992. Idaea blaesii sp. n., eine neue Sterrhinae aus Portugal (Lepidoptera: Geometridae). Mitteilungen der Münchner Entomologischen Gesellschaft, 82: 47-55.

Markl, H. \& Tautz, J., 1975.The sensitivity of hair receptors in caterpillars of Barathra brassicae (Lepidoptera: Noctuidae) to particle movement in a sound field. Journal of Comparative Physiology, 99: 79-87.

McGuffin, W. C., 1964. Setal patterns of the anterior abdominal segments of larvae of the Geometridae (Lepidoptera). Canadian Entomologist, 96: 841-849.

Reisser, H. \& Weisert, F., 1977. Eine neue Sterrha aus Spanien: Sterrha deitanaria n. sp. (Lepidoptera: Geometridae). Zeitschrift der Arbeitsgemeinschaft österreicher Entomologie, 28(4): 106-108.

Sannino, L. \& Espinosa, B., 1999. Aspetti morfologici ed etologici di Scopula turbidaria (Lepidoptera: Geometridae). Fragmenta entomologica, 31(2): 377 395.

Sannino, L. \& Espinosa, B., 2002. Note sulla morfología degli stadi preimmaginali di Idaea seriata (Lepidoptera: Geometridae). Fragmenta entomologica, 34(1): 189-206.
Singh, B. 1951. Immature stages of Indian Lepidoptera, No. 8: Geometridae. Indian Forest Records, 8(7): 67160.

Soria, S., 1987. Lepidópteros defoliadores de Quercus pyrenaica Willdenow, 1805. Ministerio de Agricultura, Pesca y Alimentación, Fuera de serie n ${ }^{\circ}$ 7. Madrid. 302 pp.

Stehr, F. W., 1987a. Techniques for collecting, rearing, preserving and studying immatures. In: F. W. Stehr (ed.). Immature insects, Tomo 1. Kendall/Hunt Publishing Company. Dubuque: 7-18.

Stehr, F. W., 1987b. Geometridae. In: F. W. Stehr (ed.). Immature insects, Tomo 1. Kendall/Hunt Publishing Company. Dubuque: 502-507.

Wiltshire, E. P., 1962. Early stages of the Old World Lepidoptera XII. Journal of the Bombay Natural History Society, 59: 778-800.

Winter, W. D., 2000. Basic Techniques for Observing and Studying Moths and Butterflies. The Lepidopterists' Society Memoir, 5. Los Ángeles. 444 pp. 